\title{
Trait Mindfulness and Emotion Regulation upon Autobiographical Memory Retrieval during Depression Remission
}

\author{
Aleksandra Eriksen Isham ${ }^{1}$ (D) $\cdot$ Adriana del Palacio-Gonzalez ${ }^{2,3}$ (D) Barbara Dritschel $^{1}$ (i)
}

Published online: 10 September 2020

(C) The Author(s) 2020

\begin{abstract}
Objectives Depression is associated with both emotion dysregulation upon retrieval of autobiographical memories and low trait mindfulness. The present study raised the question of whether these processes are related to each other and whether they may reflect a cognitive-affective vulnerability in individuals at risk of depression. The study examined emotion regulation in response to involuntary and voluntary autobiographical memories during depression remission and explored how trait mindfulness relates to such emotion regulation.

Methods The study employed a naturalistic design in which individuals with remitted depression $(n=35)$ and individuals with no history of depression $(n=32)$ completed a trait mindfulness measure and a structured memory diary where they rated state use of five emotion regulation strategies upon involuntary and voluntary autobiographical memory retrieval.

Results Individuals with remitted depression reported heightened brooding in response to autobiographical memories when memory retrieval occurred involuntarily, and trait mindfulness was low. Depression remission and higher trait mindfulness were independently associated with greater cognitive reappraisal efforts upon involuntary retrieval of autobiographical memories. Higher trait mindfulness predicted less memory suppression, irrespective of depression history and memory retrieval mode.

Conclusions The findings suggest a vulnerability in emotional processing of autobiographical memories during depression remission that is dependent on mindfulness skill level and how memories come to mind.
\end{abstract}

Keywords Mindfulness $\cdot$ State emotion regulation $\cdot$ Depression $\cdot$ Autobiographical memory $\cdot$ Naturalistic

Depression is associated with altered emotional processing of autobiographical memories. For example, individuals with acute dysphoria or depression show a bias towards retrieving negative autobiographical memories and report more intense negative emotions (i.e. fear, anger, and sadness) upon autobiographical memory retrieval, compared to individuals without depression (del Palacio-Gonzalez et al. 2017; Watson et al.

Electronic supplementary material The online version of this article (https://doi.org/10.1007/s12671-020-01494-4) contains supplementary material, which is available to authorized users.

Aleksandra Eriksen Isham

aei5@st-andrews.ac.uk

1 School of Psychology and Neuroscience, University of St Andrews, St Mary's Quad, South Street, St Andrews, Scotland KY16 9JP, UK

2 Department of Psychology and Behavioural Sciences, Aarhus University, Bartholins Allé 11, 8000 Aarhus, Denmark

3 Center on Alcohol and Drug Research, Aarhus University, Bartholins Allé 10, 8000 Aarhus, Denmark
2012). Moreover, they display impaired emotion regulation as reflected by heightened rumination, expressive suppression, and memory suppression upon retrieving autobiographical memories (del Palacio-Gonzalez et al. 2017; Watson et al. 2012).

Autobiographical memories can be retrieved either spontaneously (i.e. involuntary retrieval) or effortfully (i.e. voluntary retrieval) (e.g. Berntsen 2009). Studies investigating spontaneous thoughts and depression vulnerability have identified spontaneous cognitions, such as involuntary memories, as a potential trigger for cognitive processes associated with depression, such as rumination (for a review, see Marchetti et al. 2016). Similarly, research focusing on intrusive memories (i.e. involuntary memories of past negative events; Williams and Moulds 2007) suggests that intrusive memories may trigger rumination, which in turn predicts higher levels of depressive symptoms (Smets et al. 2012). Further, individuals vulnerable to depression (i.e. individuals with acute or remitted depression) respond to intrusive memories with greater distress, more negative emotions, and memory avoidance, 
compared to individuals with no history of depression (Newby and Moulds 2011).

Although some studies have found that dysphoria and depression are associated with more maladaptive emotional responses irrespective of memory retrieval mode (del Palacio-Gonzalez et al. 2017; Watson et al. 2012), the above findings indicate that memories that are retrieved involuntarily may present a particular challenge for individuals vulnerable to depression. However, as the studies focusing on intrusive memories (Newby and Moulds 2011; Williams and Moulds 2007) have not included comparisons of involuntarily and voluntarily retrieved memories, further research comparing emotion regulation upon involuntary and voluntary memory retrieval is needed to determine whether involuntary retrieval presents a greater challenge than voluntary retrieval.

Heightened habitual employment of maladaptive emotion regulation strategies has been identified as a cognitive vulnerability that can be observed in remitted depression as well as acute depression. Habitual rumination, or repetitive focus on one's negative affect, its causes and consequences (NolenHoeksema and Morrow 1991), is common during both acute (Watkins and Moulds 2009; Wilkinson and Goodyer 2006) and remitted depression (e.g. Aker et al. 2014; D'Avanzato et al. 2013). It may lead to increases in negative emotion, maintain or exacerbate depressed mood, and predict onset of depressive disorders (Nolen-Hoeksema 2000; NolenHoeksema et al. 1993). There is evidence to suggest that the long-term negative consequences of rumination may be driven by a subcomponent labelled brooding (i.e. passive and negative self-focus), while the long-term consequences of the subcomponent reflection (i.e. problem-solving oriented self-focus) are less clear (for a review, see Nolen-Hoeksema et al. 2008).

Elevated habitual thought suppression, or attempts to avoid thoughts that might trigger unpleasant emotions (Wegner et al. 1987), is another emotion regulation strategy that is common among individuals with acute or remitted depression (Watkins and Moulds 2009; Wenzlaff et al. 2002). Paradoxically, thought suppression may lead to an increase in unwanted thoughts (Wegner et al. 1987) and predict subsequent depressive symptoms (Rude et al. 2002). There is also evidence to suggest that both acute and remitted depression are associated with heightened use of expressive suppression (e.g. Aker et al. 2014; D'Avanzato et al. 2013), or attempts to suppress emotional expressive behaviour (Gross and Levenson 1993). High habitual use of expressive suppression is associated with experiencing less positive emotion and more negative emotion (Gross and Levenson 1997), and predicts higher subsequent depressive symptoms (Zahniser and Conley 2018).

Finally, acute depression is characterized by lowered habitual employment of cognitive reappraisal, while evidence is inconclusive for remitted depression (for a review, see
Dryman and Heimberg 2018). Cognitive reappraisal encompasses changing the meaning of a situation (e.g. reframing it as more positive) in a way that changes its emotional impact (Gross and Thompson 2007). It is considered an adaptive emotion regulation strategy, as it generally leads to effective downregulation of negative emotion (Gross 1998; Gross and John 2003).

Overall, these findings suggest that both individuals with acute depression and individuals with a history of depression regulate their emotions in ways that may increase the risk of subsequent depressive symptomatology. The persistence of habitual emotion regulation impairment during depression remission is consistent with the idea that individuals with a history of depression display cognitive-affective vulnerabilities that may put them at heightened risk of future depressive episodes (e.g. Lewinsohn et al. 1981; Teasdale 1988). However, less is known about state emotion regulation in response to everyday experiences, such as retrieval of autobiographical memories. Investigating how depression vulnerability may be reflected in such responses could shed light on important everyday contexts that may trigger depressogenic emotional responses.

A potential link between depression and emotional responses to memories may be found in mindfulness. Mindfulness refers to a mode of awareness characterized by present-moment awareness, non-judging, and non-reactivity towards inner experiences (Kabat-Zinn 2015). High trait (i.e. habitual) mindfulness is associated with lower levels of depression (for a review, see Carpenter et al. 2019). It is also associated with lower levels of habitual rumination and thought suppression (Desrosiers et al. 2013; Thompson and Waltz 2010), as well as greater employment of cognitive reappraisal (Desrosiers et al. 2013; Hanley and Garland 2014). Further, fMRI studies show that high trait mindfulness is associated with patterns of activation in the prefrontal cortex (PFC) and amygdala that are suggestive of enhanced emotion regulation (Creswell et al. 2007).

In theory, mindfulness also plays an important role in mind wandering and spontaneous cognitions (e.g. Segal et al. 2002). Given this association, it is possible that mindfulness is particularly relevant for targeting reactions to involuntary (i.e. spontaneous) memories. Recent evidence suggests that high trait mindfulness is associated with fewer intrusions of stressful events or obsessive thoughts, as well as less distressing experiences and maladaptive emotion regulation related to obsessive intrusive thoughts (Díaz et al. 2014; Emerson et al. 2018). Other evidence suggests that mindfulness meditation may enhance attentional control during sudden or rapid events, as opposed to slower, more controlled events. In particular, one study found that mindfulness and concentrative meditators were equally accurate at estimating the number of beeps when they were presented at slower rates (Valentine and Sweet 1999). However, mindfulness 
meditators outperformed concentrative meditators during a subsequent set in which beeps were presented at a faster rate, which may suggest superior attentional control during rapid or sudden events.

Given that cognitive control processes have been linked to effective emotion regulation (e.g. Hendricks and Buchanan 2016; Schmeichel et al. 2008), and the sudden nature of involuntary memories (e.g. Berntsen 2010), the above finding raises the question of whether mindfulness may be particularly helpful for targeting emotional responses to involuntary memories, as opposed to voluntary memories. In other words, the links found between mindfulness and reactions to spontaneous or intrusive cognitions raise the possibility that mindfulness may interact with memory retrieval mode (i.e. involuntary vs. voluntary) in influencing emotion regulation in response to autobiographical memories.

In the present study, we examined potential differences in state emotion regulation upon involuntary and voluntary autobiographical memory retrieval between individuals in remission from depression and individuals with no history of depression. We also examined whether trait mindfulness was associated with emotion regulation upon retrieval of autobiographical memories. We hypothesized that individuals with remitted depression would exhibit an overall greater employment of dysfunctional emotion regulation efforts upon retrieving autobiographical memories compared to individuals with no history of depression. We considered whether this effect would be stronger for involuntary memories than for voluntary memories. Further, we hypothesized that high trait mindfulness would predict lower state rumination, memory suppression, and expressive suppression, and greater cognitive reappraisal upon autobiographical memory retrieval. We expected that these relationships would be moderated by memory retrieval mode such that they would be stronger for involuntary memories compared to voluntary memories (i.e. an interaction between retrieval mode and trait mindfulness). Finally, we conducted exploratory analyses for the interaction of trait mindfulness, depression group, and memory retrieval mode.

\section{Methods}

\section{Participants}

Participants were undergraduate and postgraduate students assessed for depression. Thirty-nine individuals with no depression history (10 male) and 47 participants who met the criteria for past depression (12 male), as assessed by the MiniInternational Neuropsychiatric Interview Plus (MINI+) (Sheehan et al. 1998), completed their memory diaries $(N=$ 86). Among these participants, eight did not complete the study as instructed (e.g. failed to adhere to study protocol), and two were univariate outliers (i.e. $3 S D$ beyond mean). Further, among the individuals with a history of depression, nine also had a current depressive episode. Data from these 19 participants were not analysed. The final sample consisted of 32 individuals with no history of depression (10 male) and 35 individuals with remitted depression (10 male) $(N=67)$. The gender distributions between groups were not statistically different, $\chi^{2}(1, N=67)=.057, p=.811$. There was no age difference between groups, $t(65)=0.046, p=.964(M=$ 21.66, $S D=3.31 ; M=21.62, S D=2.92)$. All participants were students (50 undergraduates; 17 non-psychology postgraduates) at the University of St Andrews, Scotland.

Individuals with no history of depression had no previous episodes of depression, as determined by the MINI+ (Sheehan et al. 1998), whereas participants with remitted depression had at least one previous depressive episode (but no current episode). Among the participants with remitted depression, $14.29 \%$ reported one past depressive episode, whereas $82.86 \%$ reported multiple depressive episodes. Information about number of depressive episodes was missing for one participant. There was a significantly higher prevalence of other psychological disorders among participants in remission from depression $(M=0.83, S D=0.79)$ than among participants with no history of depression $(M=0.19, S D=0.47)$, $t(56.43)=-4.09, p<.001$. The most frequent comorbid diagnosis among participants with remitted depression was generalized anxiety disorder $(40 \%, n=14)$.

\section{Procedures}

We recruited participants via paper and online adverts. All participants were assessed for depression history employing the MINI+ prior to completing the memory diary and online questionnaires. We advertised the study on university bulletin boards, in weekly student emails, on student Facebook groups, and through a participant recruitment system at the university. The participants were self-selected individuals who responded to the advertisements and were fluent in English and 18 years of age or older.

Individuals who expressed interest in participating were provided with further information about the study. Individuals who agreed to participate completed an informed consent form and a demographics questionnaire and were invited to the study if they met the requirements for English fluency and age. Eligible participants received an email with a personal link that gave them access to the self-report questionnaires listed below (and one other questionnaire not analysed in the present study), in the online survey system Qualtrics. After completion of the online questionnaires, we invited the participants to meet with the first author to complete the MINI+, go over the memory diary instructions, and give materials necessary for completing the diary, including a pocket-sized notebook to record involuntary memories and a 
structured booklet with follow-up memory questions. The participants took the materials home and were asked to record memories for either up to 5 days or until they had completed seven involuntary and seven voluntary memories. After returning their memory diaries, the participants were given a debrief sheet. All participants were compensated for their participation at a rate of $£ 5 /$ hour. The local board for research ethics at the university approved the study prior to commencement.

\section{Measures}

Mini-International Neuropsychiatric Interview Plus (MINI+) The MINI+ (Sheehan et al. 1998) is a clinical interview used to diagnose psychological disorders, including depression history. It is compatible with diagnostic criteria of the International Classification of Diseases (ICD) and the Diagnostic and Statistical Manual of Mental Disorders (DSM). The sections of the MINI+ included in the present study were past and current major depressive episode (MDE), (hypo) manic episode, dysthymia, post-traumatic stress disorder (PTSD), panic disorder, alcohol abuse and dependence, and generalized anxiety disorder (GAD). To assess interrater reliability, $10 \%$ of the interviews were double-rated by two researchers trained to use the MINI+. Following independent ratings of these interviews, the interrater agreement was $87.7 \%$ for depression diagnostics and $100 \%$ for remaining diagnostics. Following discussion consensus was reached for all diagnostic conclusions.

Depression, Anxiety, Stress Scales The Depression, Anxiety, Stress Scales (DASS) (Lovibond and Lovibond 1995a) is a self-report measure that consists of 42 items across three scales measuring symptoms of depression (e.g. I felt downhearted and blue), anxiety (e.g. I was worried about situations in which I might panic and make a fool of myself), and stress (e.g. I found it difficult to relax). Each scale has 14 items corresponding to a symptom or experience, on which subjects are asked to rate the degree to which they have experienced each symptom over the past week. The DASS uses a 4-point rating scale $(0=$ did not apply to me at all, $3=$ applied to me very much or most of the time).

The DASS show good discriminant validity and successfully discriminate between depression, anxiety, and stress (Lovibond and Lovibond 1995b). The DASS depression scales show satisfactory convergent validity with the Beck Depression Inventory-II (Beck et al. 1996). In the current study, the Cronbach alphas were $.93, .89$, and .91 for the depression, anxiety, and stress subscales, respectively.

Ruminative Response Scale We employed the Ruminative Response Scale (RRS) (Nolen-Hoeksema and Morrow 1991) to assess dispositional brooding and reflection. The scale is a self-report measure that includes 22 statements that describe ruminative responses to depressed mood (e.g. Think: "Why do I always react this way?" (brooding); analyse recent events to try to understand why you are depressed (reflection)). It uses a 4-point rating scale $(1=$ almost never, $4=$ almost always) on which subjects rate the degree to which they engage in different responses. The RRS can be divided into subscales of reflection and brooding, composed of 5 items each (Treynor et al. 2003). The subscales have shown adequate convergent and discriminant validity (Schoofs et al. 2010). In the current study, the scales exhibited good internal consistency ( $\alpha=.91, \alpha=.75$, and $\alpha=.75$ for the full scale, reflection subscale, and brooding subscale, respectively).

Emotion Regulation Questionnaire The Emotion Regulation Questionnnaire (ERQ) (Gross and John, 2003) measures dispositional cognitive reappraisal and expressive suppression. The cognitive reappraisal subscale consists of six items referring to cognitive reappraisal responses (e.g. I control my emotions by changing the way I think about the situation I'm in), whereas the expressive suppression subscale consists of four items referring to expressive suppression responses (e.g. I control my emotions by not expressing them). Subjects are asked to rate to which degree they engage in each response, using a 7-point rating scale $(1=$ strongly disagree, $7=$ strongly agree). The scales show adequate convergent and discriminant validity, and factor analyses support the questionnaire's two-factor structure (Gross and John 2003). In the current study, the scales showed good internal consistency ( $\alpha=.86$ and $\alpha=.83$ for cognitive reappraisal and expressive suppression, respectively).

White Bear Suppression Inventory The White Bear Suppression Inventory (WBSI) (Wegner and Zanakos 1994) consists of 15 items assessing thought suppression responses (e.g. There are things that I try not to think about). Subjects rate the degree to which they engage in each response, using a 5 -point rating scale $(1=$ strongly disagree, $5=$ strongly agree). The WBSI has shown good predictive and convergent validity (Wegner and Zanakos 1994) and exhibited high internal consistency $(\alpha=.89)$ in the current study.

Five Facet Mindfulness Questionnaire We measured trait mindfulness with the Five Facet Mindfulness Questionnaire (FFMQ) (Baer et al. 2006). This self-report consists of 39 statements (e.g. I perceive my feelings and emotions without having to react to them) across five facets on which subjects rate their agreement using a 5 -point scale $(1=$ never or very rarely true, $5=$ very often or always true). Baer et al. (2006) found that a sample of meditators had higher FFMQ scores compared to a student sample, a community sample, and a sample of highly educated individuals, suggesting that the FFMQ measures mindfulness skills across individuals with 
different backgrounds and experience with mindfulness practice. In the current study, the total scale exhibited a Cronbach's alpha of .87 .

Autobiographical Memory Diary We employed a structured memory diary to collect involuntary and voluntary (wordcued) memories and corresponding emotion regulation. The structure of the diary was based on a well-established diary methodology first developed by Berntsen and Hall (2004) and later modified by del Palacio-Gonzalez et al. (2017). The instructions for the diary were adjusted for the current study, as indicated below.

The participants were given a pocket-sized notebook and a structured diary booklet. They were asked to record up to seven involuntary and seven voluntary memories over a period of up to 5 days. The participants followed three main steps to complete their diaries. The first step was to record involuntary memories immediately upon retrieval in their pocket-sized notebook. The participants rated each involuntary memory along several dimensions, including mood before the memory appeared $(-2=$ very bad to $2=$ very good $)$, the emotional intensity experienced upon memory retrieval (fear, happiness, sadness, anger, and 'other', each rated from $1=$ not at all to $5=$ a great deal), the employment of five emotion regulation strategies and state mindfulness (cognitive reappraisal, reflection, brooding, memory suppression, expressive suppression, and non-reactivity; $1=$ not at all to $5=$ a great deal), and valence of the remembered event at the time it happened $(-2=$ very negative to $+2=$ very positive). The questions assessing employment of emotion regulation upon memory retrieval were the same as employed by del Palacio-Gonzalez et al. (2017) in their memory diary study. These were in turn based on items from the ERQ, the WBSI, and the RRS, specifically: I changed the way I was thinking about the situation (cognitive reappraisal); I analysed the events to try to understand my feelings (reflection); I thought: Why do I always react this way? (brooding); I tried not to keep thinking about it (memory suppression); and I controlled my emotion by not expressing it (expressive suppression). The employment of single items to assess emotion regulation in naturalistic studies has been found to result in reliable and valid assessments (Ong et al. 2006).

As a second step, the participants transferred their notebook ratings for the involuntary memories to a structured memory booklet later in the day. At that point, the participants rated additional characteristics of the involuntary memories, including specificity, and how central the memory was for the individual's identity. For the purpose of the present study, only emotion regulation questions were analysed.

As a third and final step, immediately after completing the follow-up questions for each involuntary memory, the participants were asked to uncover a cue word in the diary booklet and to retrieve a memory in response to the cue (i.e. a voluntary memory). The cues were taken from del PalacioGonzalez et al. (2017) and consisted of the words book, rain, happy, cleaning, flowers, sad, and family. These cues match the content of involuntary memories activated in daily life (see also Berntsen and Hall 2004). The participants then rated the voluntary memory across the same dimension as the involuntary memories (e.g. emotion regulation).

The participants were told that they could exclude memories that they felt were too intimate or embarrassing to record. An important advantage of this memory diary is that memories are reported and rated immediately rather than retrospectively. The memory diary also aims to minimize selection bias of memories by including only the first two memories per day (Berntsen 2009). However, in the present study, the participants were allowed to record the first three memories of the day and the period of the diary was restricted to 5 days.

Although we had restricted the memory diary period to 5 days, some participants spent longer than this. Two participants spent 13 and 31 days and we excluded them from our analyses. The remaining participants spent less than 10 days. Due to the deviation from the instructions, we reviewed the content of randomly selected diaries that were completed in more than 5 days and compared them with diaries that were completed in up to 5 days. We did not find any consistent differences in content (i.e. themes and richness of memory descriptions). Therefore, in the end, the participants who recorded their memories within 10 days were included in our data analyses. Given that participants could record zero to three memories per day, they finished the diary at different rates $(M=5.18$ days; $S D=1.95)$. Likewise, given that the participants could record up to seven involuntary and seven voluntary memories (max. 14 total), the number of memories per participant varied $(M=12.75$ memories; $S D=2.64)$. The groups did not differ significantly in their completion rates, $t(62)=1.77, p=.081$ or number of memories reported, $t(65)=$ $0.722, p=.775$.

\section{Data Analyses}

We employed bootstrapping when conducting group comparisons as this is considered a robust method for dealing with non-normality and heteroskedasticity (Field 2013). All confidence intervals (CIs) for group differences are bias-corrected (BCa) CIs based on 1000 bootstrap samples and are considered statistically significant when they do not include zero.

We examined the hypotheses concerning the relationship between trait mindfulness and emotion regulation upon memory retrieval employing multilevel modelling (MLM). The models held 854 observations nested within 67 individuals. A study that used simulation to estimate sufficient sample sizes for MLM suggests that a higher-level sample (i.e. participants in the present study) of 50 or more produces accurate 
regression coefficients and variance components, and acceptable estimates of standard errors (Maas and Hox 2005). Level 1 in the models consisted of memory-level variables, including repeated measures of memory emotion regulation nested within participants as well as retrieval mode. Level 2 consisted of trait mindfulness and depression group. We selected the autoregressive model (AR1) as the covariance structure for the repeated measures. For the remaining parameters, we selected the variance components (VC) covariance structure.

We conducted separate models to predict each of the five daily emotion regulation strategies assessed: brooding, reflection, memory suppression, expressive suppression, and cognitive reappraisal. The models were tested from simpler (model 1: unconditional) to more complex (model 2: simple predictors and model 3: simple predictors and cross-level interactions) in accordance with best-practice recommendations (Aguinis et al. 2013). The simple predictors in models 2 and 3 included trait mindfulness (continuous; grand mean centred), memory retrieval mode (involuntary $=1$; voluntary $=2$ ), and group (no history of depression $=0$; remitted depression $=1$ ).

We evaluated the models' improvement of fit hierarchically, by assessing a significant decrease in $-2 \log$ likelihood (2LL) when adding parameters (i.e. simple predictors, random slopes, and cross-level interactions). Including simple predictors (model 2) significantly improved the fit for brooding, cognitive reappraisal, and memory suppression, but not for reflection and expressive suppression compared to the unconditional models. In model 2, random slopes for trait mindfulness improved the goodness of fit for cognitive reappraisal and brooding but not for memory suppression. In a final step, we included cross-level interactions (model 3), which improved model fit for brooding and cognitive reappraisal, but not memory suppression. Therefore, model 2 without random slopes is interpreted for memory suppression and expressive suppression, whereas model 3 with random slopes for trait mindfulness is interpreted for brooding and cognitive reappraisal. We calculated Cohen's $d$ for significant effects. All analyses were conducted in SPSS Version 25.

\section{Results}

\section{Group Differences in Symptoms and Trait Variables}

As shown in Table 1, participants with remitted depression reported significantly higher depression and stress symptoms on the DASS than participants with no depression history. Participants with remitted depression also reported significantly lower trait mindfulness than participants with no history of depression, as well as greater dispositional thought suppression, brooding, reflection, and overall rumination. There were no significant between-group differences in cognitive reappraisal or expressive suppression.

\section{Simple Predictors of Daily Emotion Regulation}

Depression Group Table 2 shows the means and SDs in the use of daily emotion regulation upon memory retrieval for individuals with and without a history of depression. Group differences were examined using MLM. Contrary to expectations, individuals with remitted depression reported significantly greater employment of cognitive reappraisal, $d=.31$ (Table 3, model 3) upon memory retrieval compared to individuals without a history of depression. Depression remission did not predict overall employment of other emotion regulation strategies upon memory retrieval.

Trait Mindfulness Our hypothesis regarding an overall relationship between trait mindfulness and emotion regulation upon memory retrieval was supported for two strategies. As expected, higher trait mindfulness was significantly related to higher cognitive reappraisal, $d=.36$, and lower memory suppression, $d=-.51$ (see Table 3 , models 2 and 3 ). Contrary to our expectations, higher trait mindfulness also predicted higher brooding, $d=.26$.

Memory Retrieval Mode Involuntary retrieval was related to greater memory suppression compared to voluntary memories, $d=-.28$ (Table 3 ; model 2), in line with previous findings (del Palacio-Gonzalez et al. 2017). The retrieval mode did not predict the employment of other emotion regulation strategies upon memory retrieval.

\section{Moderation Effects Predicting Daily Emotion Regulation}

Two-Way Interactions We hypothesized that the memory retrieval mode would moderate the effect of depression history on emotion regulation upon memory retrieval, as assessed by a group * retrieval interaction. There was a significant group * retrieval interaction predicting cognitive reappraisal, $d=-.29$ (see Table 3; model 3). The follow-up of this interaction indicated that participants with remitted depression reported significantly greater employment of cognitive reappraisal compared to individuals with no depression history, when retrieving involuntary memories, $b=0.31, t(116.24)=2.67, p=$ .009. There were no group differences in cognitive reappraisal during voluntary retrieval, $b=-0.00, t(137.52)=-0.052, p=$ .958 .

We also hypothesized that the relationship between trait mindfulness and emotion regulation upon memory retrieval would be moderated by the memory retrieval mode (mindfulness * retrieval interactions). The interactions were significant for brooding, $d=.26$, and cognitive reappraisal, $d=.34$ 
Table 1 Differences between never-depressed $(n=32)$ and remitted-depressed $(n=35)$ individuals in symptoms, trait mindfulness, and dispositional emotion regulation

\begin{tabular}{|c|c|c|c|c|c|c|c|}
\hline & \multicolumn{2}{|l|}{ Never depressed } & \multicolumn{2}{|c|}{ Remitted depressed } & \multirow[t]{2}{*}{$t(65)$} & \multirow[t]{2}{*}{$p$} & \multirow{2}{*}{$\begin{array}{l}\text { Mean diff. bootstrap BCa } \\
95 \% \text { CI }\end{array}$} \\
\hline & $M$ & $S D$ & $M$ & $S D$ & & & \\
\hline DASS depression & $\begin{array}{l}5.06 \\
{[3.10,7.16]}\end{array}$ & 5.54 & $\begin{array}{l}12.86 \\
{[10.12,15.68]}\end{array}$ & 8.07 & -4.64 & $<.001$ & {$[-11.50,-4.31]$} \\
\hline DASS anxiety & $\begin{array}{l}6.53 \\
{[4.50,8.99]}\end{array}$ & 5.85 & $\begin{array}{l}9.57 \\
{[7.30,12.08]}\end{array}$ & 7.92 & -1.77 & .081 & {$[-6.46,0.36]$} \\
\hline DASS stress & $\begin{array}{l}9.69 \\
{[7.28,12.40]}\end{array}$ & 7.32 & $\begin{array}{l}16.46 \\
{[13.65,19.45]}\end{array}$ & 8.85 & -3.39 & .001 & {$[-10.99,-2.43]$} \\
\hline FFMQ & $\begin{array}{l}121.22 \\
{[116.48,125.90]}\end{array}$ & 15.13 & $\begin{array}{l}111.51 \\
{[105.99,116.82]}\end{array}$ & 16.07 & 2.54 & .014 & {$[2.59,16.88]$} \\
\hline RRS total & $\begin{array}{l}42.03 \\
{[37.57,46.27]}\end{array}$ & 11.67 & $\begin{array}{l}50.17 \\
{[47.09,53.64]}\end{array}$ & 9.72 & -3.11 & .003 & {$[-13.59,-3.09]$} \\
\hline RRS brooding & $\begin{array}{l}9.44 \\
{[8.33,10.55]}\end{array}$ & 3.04 & $\begin{array}{l}11.20 \\
{[10.30,12.12]}\end{array}$ & 2.79 & -2.47 & .016 & {$[-3.21,-0.39]$} \\
\hline RRS reflection & $\begin{array}{l}9.88 \\
{[8.57,11.09]}\end{array}$ & 3.43 & $\begin{array}{l}11.46 \\
{[10.38,12.57]}\end{array}$ & 3.11 & -1.98 & .052 & {$[-3.20,-0.09]$} \\
\hline ERQ expressive suppression & $\begin{array}{l}13.63 \\
{[11.16,16.09]}\end{array}$ & 6.58 & $\begin{array}{l}15.31 \\
{[13.78,16.94]}\end{array}$ & 4.51 & -1.21 & .230 & {$[-4.38,1.30]$} \\
\hline ERQ cognitive reappraisal & $\begin{array}{l}27.91 \\
{[25.42,30.07]}\end{array}$ & 6.94 & $\begin{array}{l}25.89 \\
{[23.64,27.96]}\end{array}$ & 6.42 & 1.24 & .220 & {$[-1.13,5.11]$} \\
\hline WBSI thought suppression & $\begin{array}{l}47.03 \\
{[42.82,50.97]}\end{array}$ & 12.17 & $\begin{array}{l}53.15 \\
{[48.96,56.87]}\end{array}$ & 10.5 & -2.19 & .032 & {$[-11.53,-1.19]$} \\
\hline
\end{tabular}

All confidence intervals (CIs) are BCa 95\% CIs based on 1000 bootstrap samples

DASS Depression, Anxiety, Stress Scales, ERQ Emotion Regulation Questionnaire, FFMQ Five Facet Mindfulness Questionnaire, RRS Ruminative Response Scale, WBSI White Bear Suppression Inventory

(Table 3; model 3). For cognitive reappraisal, follow-up analyses of the interaction indicated that higher trait mindfulness significantly predicted higher cognitive reappraisal during involuntary retrieval, $b=0.02, t(22.56)=2.50, p=.020$, but not during voluntary retrieval, $b=-0.00, t(48.33)=-.193, p=$ .847 .

The above significant two-way interaction for brooding was further explained by a significant trait mindfulness $*$ retrieval $*$ group interaction, $d=.36$, as was the mindfulness $*$ group interaction predicting brooding, $d=-.45$ (Table 3 ; model 3). The three-way interaction is explained below.
Three-Way Interactions To follow-up to the significant trait mindfulness $*$ retrieval $*$ group interaction, we employed Preacher's web utility (Preacher et al. 2003) to determine regions of significance. The results suggested that trait mindfulness moderated the relationship between depression and brooding for involuntary memories. At lower levels of trait mindfulness (i.e. FFMQ score below 116), remitted depression predicted greater brooding upon involuntary memory retrieval (when trait mindfulness $=115.70, b=0.22, p=.050$ ). At moderate levels of trait mindfulness (i.e. FFMQ scores between 116 and 139), remitted depression did not predict
Table 2 Emotion regulation upon involuntary and voluntary autobiographical memory retrieval in never-depressed $(n=$ $32)$ and remitted-depressed ( $n=$ 35 ) individuals

\begin{tabular}{|c|c|c|c|c|c|c|c|c|}
\hline \multirow[b]{3}{*}{ State emotion regulation } & \multicolumn{4}{|c|}{ Never depressed } & \multicolumn{4}{|c|}{ Remitted-depressed } \\
\hline & \multicolumn{2}{|c|}{ Involuntary } & \multicolumn{2}{|c|}{ Word cued } & \multicolumn{2}{|c|}{ Involuntary } & \multicolumn{2}{|c|}{ Word cued } \\
\hline & $M$ & $S D$ & $M$ & $S D$ & $M$ & $S D$ & $M$ & $S D$ \\
\hline Brooding & 1.42 & 0.46 & 1.29 & 0.57 & 1.65 & 0.56 & 1.42 & 0.53 \\
\hline Memory suppression & 1.85 & 0.76 & 1.73 & 0.71 & 2.09 & 0.77 & 1.70 & 0.60 \\
\hline Reflection & 1.90 & 0.69 & 1.99 & 0.71 & 2.12 & 0.58 & 1.96 & 0.72 \\
\hline Cognitive reappraisal & 1.44 & 0.50 & 1.38 & 0.39 & 1.65 & 0.48 & 1.36 & 0.42 \\
\hline Expressive suppression & 1.79 & 0.97 & 1.72 & 0.83 & 1.90 & 0.79 & 1.75 & 0.86 \\
\hline
\end{tabular}




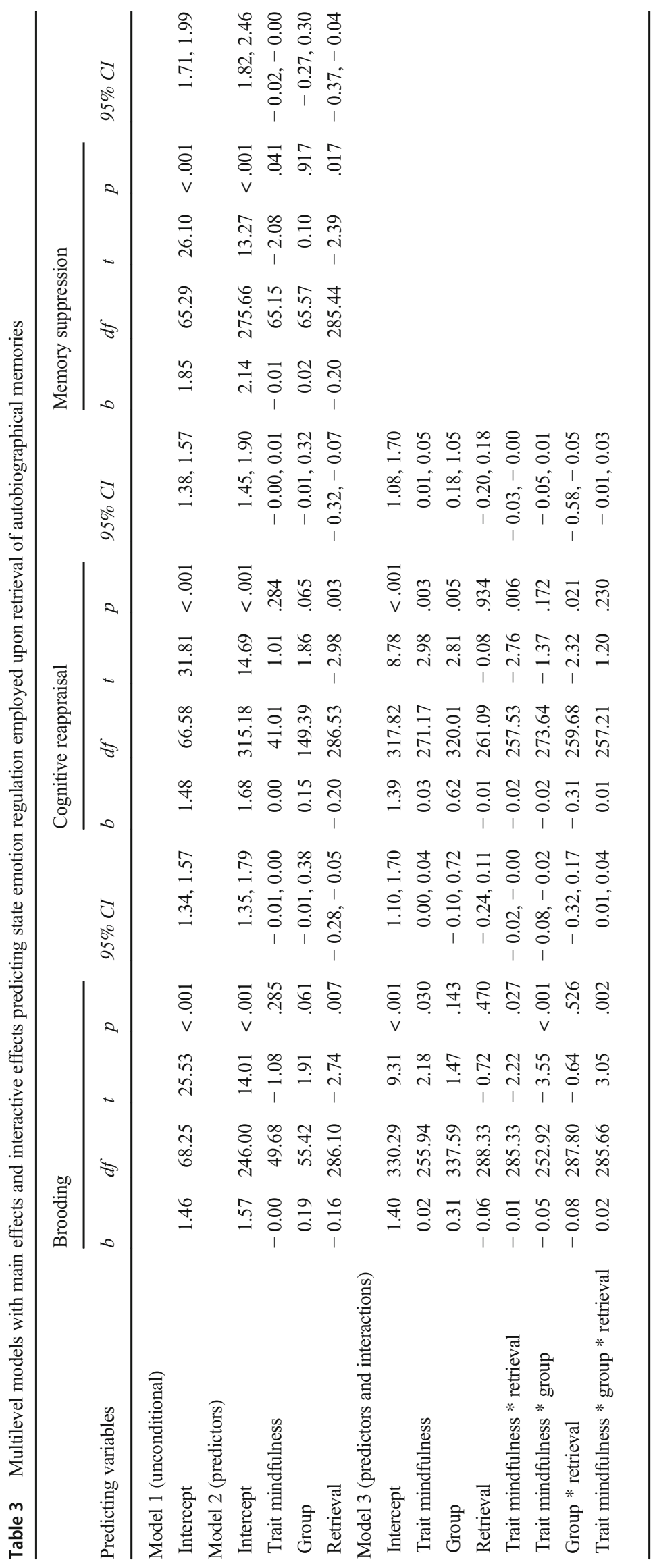


brooding upon involuntary memory retrieval. Finally, at high levels of trait mindfulness (i.e. FFMQ scores above 139), participants in remission from depression reported less brooding upon involuntary memory retrieval compared to participants with no history of depression (when trait mindfulness = $139.45, b=-0.42, p=.050$ ). For voluntary memories, remitted depression did not predict brooding, irrespective of trait mindfulness level.

\section{Supplementary Analyses: Effects of Depression Symptoms and Memory Valence}

Elevated depression symptoms are not uncommon during depression remission (e.g. Fava et al. 2007). Participants with remitted depression in the current study reported significantly higher depression scores than participants without a history of depression. Therefore, we examined whether the effects found in the present study were driven by high depression scores in the depression remission group, as opposed to an underlying vulnerability during remission. We conducted supplemental analyses controlling for depression symptoms in betterfitting multilevel models (Table 3, model 2 and model 3). For brooding and cognitive reappraisal, all statistically significant effects from the main analyses remained significant after controlling for depression symptoms. For memory suppression, all statistically significant effects from the main analyses remained significant, except trait mindfulness $(p=.083)$. That is, when the effect of depression symptoms was controlled for, the effect of trait mindfulness on memory suppression was no longer statistically significant.

Lastly, we examined whether the emotion regulation in our main analyses was driven by memory valence. For brooding and cognitive reappraisal, all statistically significant effects from the main analyses (Table 3) remained significant after controlling for memory valence. For memory suppression, all significant effects shown in Table 3 remained significant, except trait mindfulness $(p=.170)$. That is, when the effect of memory valence was controlled for, the effect of trait mindfulness on memory suppression was no longer statistically significant. This might suggest that the relationship between trait mindfulness and memory suppression is valence dependent.

\section{Discussion}

The aims of the present study were twofold. First, we wanted to assess whether depression remission predicted emotion regulation upon involuntary and voluntary retrieval of autobiographical memories. Second, we investigated the relationship between trait mindfulness and emotion regulation employed upon autobiographical memory retrieval in individuals with and without a history of depression. We also explored the potential moderating role of memory retrieval mode in the relationships between mindfulness, past depression, and emotion regulation upon memory retrieval.

We found that the relationship between depression remission and emotion regulation upon memory retrieval was moderated by memory retrieval mode in predicting the use of brooding and cognitive reappraisal. Individuals with remitted depression reported higher employment of cognitive reappraisal during involuntary retrieval but not during voluntary retrieval, compared to individuals with no depression history. Remission from depression was also associated with greater brooding upon involuntary memory retrieval, but only if trait mindfulness was low. When trait mindfulness was higher, participants with remitted depression no longer reported greater brooding than participants with no history of depression.

The moderated relationship between depression remission and brooding indicates that experiencing involuntary memories may foster a heightened use of brooding among individuals with remitted depression who exhibit low trait mindfulness. The fact that this pattern remained after controlling for depression symptom severity suggests that it reflects an underlying vulnerability as opposed to a concomitant phenomenon of acute depressive symptoms. The findings also indirectly suggest that high trait mindfulness may protect against such vulnerability.

The positive relationship between depression remission and cognitive reappraisal upon involuntary memory retrieval is consistent with other studies in which dysphoric individuals employed numerically (albeit not significantly) greater state cognitive reappraisal compared to controls (del PalacioGonzalez et al. 2017; Ehring et al. 2010). On the other hand, it is in contrast with the current study's and previous studies' findings for trait levels of cognitive reappraisal. Specifically, in the present study, we found that individuals with and without a history of depression did not differ in their trait use of cognitive reappraisal, while previous studies have found either no difference or lowered trait cognitive reappraisal in individuals in remission from depression (for a review, see Dryman and Heimberg 2018).

These inconsistencies between trait and state cognitive reappraisal may highlight the importance of considering contextual factors for emotion regulation (e.g. state vs. trait emotion regulation) when investigating emotion regulation and depression vulnerability. Another important consideration is that, although cognitive reappraisal is generally regarded as an adaptive emotion regulation strategy (Gross and John 2003), the use of this strategy during depression does not directly point to successful emotion regulation. In particular, there is evidence that individuals with remitted depression fail to show an inverse relationship between activation in the ventromedial prefrontal cortex (VMPFC) and amygdala during cognitive reappraisal, compared to healthy individuals (Johnstone et al. 2007). This lack of inverse association between 
activation in the VMPFC and amygdala may indicate less successful emotion regulation, as the medial PFC has been implicated in amygdala downregulation. In our study, we did not assess the effects of employing cognitive reappraisal upon memory retrieval. Therefore, it is unclear whether the higher use of cognitive reappraisal during depression remission reflects efficient emotion regulation or, alternatively, a vulnerability characterized by unsuccessful reappraisal efforts.

Beyond the effects of depression remission, we found that high trait mindfulness was related to state emotion regulation in two ways: lower memory suppression and higher cognitive reappraisal. Regarding memory suppression, the finding is consistent with studies that have found an inverse relationship between trait mindfulness and thought suppression at the trait level (Thompson and Waltz 2010), as well as with conceptualizations of trait mindfulness (e.g. non-judging and non-reactivity; Baer et al. 2006). From this perspective, our data suggest that more mindful individuals may be more likely to acknowledge and accept their autobiographical memories, rather than trying to suppress them. As greater employment of thought suppression predicts higher levels of subsequent depression (Rude et al. 2002), high trait mindfulness and the associated lower suppression of autobiographical memories may reduce the risk of future depression. It is important to note that the effect of trait mindfulness on memory suppression did not remain significant when controlling for memory valence. This may suggest a valencedependent effect for this emotion regulation strategy that could be explored more systematically in future studies.

In the case of cognitive reappraisal, we found that more mindful individuals, irrespective of depression history, employed greater cognitive reappraisal upon retrieving involuntary, but not voluntary, memories compared to less mindful individuals. This finding is partly consistent with previous studies suggesting a positive relationship between trait mindfulness and dispositional cognitive reappraisal (Desrosiers et al. 2013; Hanley and Garland 2014), but underscores the importance of understanding the nature of the cognitions triggering emotion regulation. Specifically, our finding suggests that trait mindfulness has a greater influence on cognitive reappraisal for memories that occur spontaneously than memories that occur voluntarily. Along with our findings for brooding, the above finding lends initial support to the idea that mindfulness may be particularly useful for targeting emotional responses to involuntary or spontaneous cognition (Díaz et al. 2014; Emerson et al. 2018) as opposed to voluntary or effortful cognition.

Our findings have implications for vulnerability models of depression. Spontaneous cognition has been proposed to trigger cognitive processes that are associated with depressogenic vulnerability (e.g. Marchetti et al. 2016). One of the suggested routes of vulnerability is that spontaneous cognition activates maladaptive emotion regulation, such as rumination. Such notion is best tested if emotion regulation is compared during the retrieval of spontaneous versus non-spontaneous cognition, which was done in the current study. Present findings do lend some support to the idea that spontaneous cognition fosters depressogenic processes in individuals at risk of depression, especially when they display low habitual use of mindfulness. Our findings suggest that such vulnerability may be characterized by a heightened reliance on brooding when autobiographical memories are retrieved involuntarily, irrespective of memory valence.

\section{Limitations and Future Research}

There were limitations to our study that should be considered when interpreting its results. Our sample size was relatively small, and we collected fewer memories than in previous autobiographical memory studies (typically 20 or more). Further, our sample was drawn exclusively from a student population, which decreases the generalizability of our findings to clinical settings. Future studies should test whether our findings are replicated in larger, clinical samples.

Another limitation relates to the way voluntary memories were cued. We employed a word-cue paradigm to maximize the distinction between voluntary and involuntary memories (see del Palacio-Gonzalez et al. 2017 for more discussion of the method). However, using word cues to elicit autobiographical memories may not be representative of everyday experiences of voluntary memories (Rasmussen et al. 2014). Future studies should test whether our findings are replicated for voluntary memories elicited in everyday contexts (e.g. in response to social interactions).

In the present study, we examined overall level of mindfulness skill in relation to a selection of emotion regulation strategies. Future studies should also investigate how specific facets of mindfulness relate to daily use of emotion regulation strategies, as there is evidence that certain facets may better predict emotion regulation than others (Petrocchi and Ottaviani 2016). This may be particularly important for reflection and expressive suppression, for which we did not find any relationship with overall trait mindfulness. In the present study, sample size did not allow this question to be examined. Future studies could also explore whether mindfulness-based interventions influence state emotion regulation upon retrieval of autobiographical memories.

Finally, since the measures of emotion regulation employed upon memory retrieval consisted of only one item per emotion regulation strategy, we were not able to capture all the conceptual richness of the emotion regulation strategies assessed. Future research could incorporate more substantial assessments of state emotion regulation strategies. 
Notwithstanding these limitations, the findings in the present study provide insights into everyday emotion regulation impairments associated with depression vulnerability, and the potential protective role of mindfulness for such impairments. They call for further research in the field intersecting autobiographical memory, emotion regulation, mindfulness, and depression prevention.

Acknowledgements We thank Nina Udvardi-Lakos for assistance with double-rating of clinical interviews in the present study.

Contributions AEI: designed and executed the study, analysed the data, and wrote the first full draft of the paper. APG: collaborated with the design, data analyses, and writing of the study. BD: collaborated with the design, data analyses, and writing of the study. All authors approved the final version of the manuscript for submission.

Funding This work was supported by the Danish National Research Foundation (grant DNRF89: APG) and the University of St Andrews (AEI and BD).

Data Availability The raw data underpinning this study cannot be made publicly available due to ethical concerns since there is no agreement from the participants to share their anonymised data. Study materials are attached as supplementary materials.

\section{Compliance with Ethical Standards}

Conflict of Interest The authors declare that they have no conflicts of interest.

Informed Consent Informed consent was obtained from all individual participants. The participants were informed that participation in the study was voluntary.

Ethical Approval The local board for research ethics at the University of St Andrews approved the study prior to commencement.

Open Access This article is licensed under a Creative Commons Attribution 4.0 International License, which permits use, sharing, adaptation, distribution and reproduction in any medium or format, as long as you give appropriate credit to the original author(s) and the source, provide a link to the Creative Commons licence, and indicate if changes were made. The images or other third party material in this article are included in the article's Creative Commons licence, unless indicated otherwise in a credit line to the material. If material is not included in the article's Creative Commons licence and your intended use is not permitted by statutory regulation or exceeds the permitted use, you will need to obtain permission directly from the copyright holder. To view a copy of this licence, visit http://creativecommons.org/licenses/by/4.0/.

\section{References}

Aguinis, H., Gottfredson, R. K., \& Culpepper, S. A. (2013). Best-practice recommendations for estimating cross-level interaction effects using multilevel modeling. Journal of Management, 39(6), 1490-1528. https://doi.org/10.1177/0149206313478188.

Aker, M., Harmer, C., \& Landrø, N. I. (2014). More rumination and less effective emotion regulation in previously depressed women with preserved executive functions. BMC Psychiatry, 14(1), 334. https:// doi.org/10.1186/s12888-014-0334-4.

Baer, R. A., Smith, G. T., Hopkins, J., Krietemeyer, J., \& Toney, L. (2006). Using self-report assessment methods to explore facets of mindfulness. Assessment, 13(1), 27-45. https://doi.org/10.1177/ 1073191105283504.

Beck, A. T., Steer, R. A., \& Brown, G. K. (1996). Manual for the Beck depression inventory-II. San Antonio: Psychological Corporation.

Berntsen, D. (2009). Involuntary autobiographical memories: an introduction to the unbidden past. Cambridge: Cambridge University Press.

Berntsen, D. (2010). The unbidden past: involuntary autobiographical memories as a basic mode of remembering. Current Directions in Psychological Science, 19(3), 138-142. https://doi.org/10.1177/ 0963721410370301.

Berntsen, D., \& Hall, N. M. (2004). The episodic nature of involuntary autobiographical memories. Memory and Cognition, 32(5), 789803. https://doi.org/10.3758/BF03195869.

Carpenter, J. K., Conroy, K., Gomez, A. F., Curren, L. C., \& Hofmann, S. G. (2019). The relationship between trait mindfulness and affective symptoms: a meta-analysis of the Five Facet Mindfulness Questionnaire (FFMQ). Clinical Psychology Review, 74, 101785. https://doi.org/10.1016/j.cpr.2019.101785.

Creswell, J. D., Way, B. M., Eisenberger, N. I., \& Lieberman, M. D. (2007). Neural correlates of dispositional mindfulness during affect labeling. Psychosomatic Medicine, 69(6), 560-565. https://doi.org/ 10.1097/PSY.0b013e3180f6171f.

D’Avanzato, C., Joormann, J., Siemer, M., \& Gotlib, I. H. (2013). Emotion regulation in depression and anxiety: examining diagnostic specificity and stability of strategy use. Cognitive Therapy and Research, 37(5), 968-980. https://doi.org/10.1007/s10608-0139537-0.

del Palacio-Gonzalez, A., Berntsen, D., \& Watson, L. A. (2017). Emotional intensity and emotion regulation in response to autobiographical memories during dysphoria. Cognitive Therapy and Research, 41(4), 530-542. https://doi.org/10.1007/s10608-0179841-1.

Desrosiers, A., Vine, V., Klemanski, D. H., \& Nolen-Hoeksema, S. (2013). Mindfulness and emotion regulation in depression and anxiety: common and distinct mechanisms of action. Depression and Anxiety, 30(7), 654-661. https://doi.org/10.1002/da.22124.

Díaz, N. S. R., Jiménez, Ó. J., \& Lopes, P. N. (2014). The role of mindfulness in coping with recollections of acute stressors: a laboratory study. Psicothema, 26(4), 505-510. https://doi.org/10.7334/ psicothema2014.71.

Dryman, M. T., \& Heimberg, R. G. (2018). Emotion regulation in social anxiety and depression: a systematic review of expressive suppression and cognitive reappraisal. Clinical Psychology Review, 65, 1742. https://doi.org/10.1016/j.cpr.2018.07.004.

Ehring, T., Tuschen-Caffier, B., Schnülle, J., Fischer, S., \& Gross, J. J. (2010). Emotion regulation and vulnerability to depression: spontaneous versus instructed use of emotion suppression and reappraisal. Emotion, 10(4), 565-575.563. https://doi.org/10.1037/a0019010.

Emerson, L. M., Heapy, C., \& Garcia-Soriano, G. (2018). Which facets of mindfulness protect individuals from the negative experiences of obsessive intrusive thoughts? Mindfulness, 9(4), 1170-1180. https://doi.org/10.1007/s12671-017-0854-3.

Fava, G. A., Ruini, C., \& Belaise, C. (2007). The concept of recovery in major depression. Psychological Medicine, 37(3), 307-317. https:// doi.org/10.1017/S0033291706008981.

Field, A. (2013). Discovering statistics using IBM SPSS statistics (4th ed.). Thousand Oaks: Sage.

Gross, J. J. (1998). Antecedent-and response-focused emotion regulation: Divergent consequences for experience, expression, and physiology. Journal of Personality and Social Psychology, 74(1), 224-237. https://doi.org/10.1037/0022-3514.74.1.224. 
Gross, J. J., \& John, O. P. (2003). Individual differences in two emotion regulation processes: implications for affect, relationships, and wellbeing. Journal of Personality and Social Psychology, 85(2), 348362. https://doi.org/10.1037/0022-3514.85.2.348.

Gross, J. J., \& Levenson, R. W. (1993). Emotional suppression: physiology, self-report, and expressive behavior. Journal of Personality and Social Psychology, 64(6), 970-986. https://doi.org/10.1037/ 0022-3514.64.6.970.

Gross, J. J., \& Levenson, R. W. (1997). Hiding feelings: the acute effects of inhibiting negative and positive emotion. Journal of Abnormal Psychology, 106(1), 95-103. https://doi.org/10.1037//0021-843X. 106.1.95.

Gross, J. J., \& Thompson, R. A. (2007). Emotion regulation: conceptual foundations. Handbook of emotion regulation (pp. 3-24). New York City: The Guilford Press.

Hanley, A. W., \& Garland, E. L. (2014). Dispositional mindfulness covaries with self-reported positive reappraisal. Personality and Individual Differences, 66, 146-152. https://doi.org/10.1016/j.paid. 2014.03.014.

Hendricks, M. A., \& Buchanan, T. W. (2016). Individual differences in cognitive control processes and their relationship to emotion regulation. Cognition and Emotion, 30(5), 912-924. https://doi.org/10. 1080/02699931.2015.1032893.

Johnstone, T., van Reekum, C. M., Urry, H. L., Kalin, N. H., \& Davidson, R. J. (2007). Failure to regulate: counterproductive recruitment of top-down prefrontal-subcortical circuitry in major depression. Journal of Neuroscience, 27(33), 8877-8884. https://doi.org/10. 1523/JNEUROSCI.2063-07.2007.

Kabat-Zinn, J. (2015). Mindfulness. Mindfulness, 6(6), 1481-1483. https://doi.org/10.1007/s12671-015-0456-x.

Lewinsohn, P. M., Steinmetz, J. L., Larson, D. W., \& Franklin, J. (1981). Depression-related cognitions: antecedent or consequence? Journal of Abnormal Psychology, 90(3), 213-219. https://doi.org/10.1037/ 0021-843X.90.3.213.

Lovibond, S. H., \& Lovibond, P. F. (1995a). Manual for the depression anxiety stress scales (2nd ed.). Sydney: Psychology Foundation.

Lovibond, P. F., \& Lovibond, S. H. (1995b). The structure of negative emotional states: comparison of the Depression Anxiety Stress Scales (DASS) with the Beck Depression and Anxiety Inventories. Behaviour Research and Therapy, 33(3), 335-343. https://doi.org/ 10.1016/0005-7967(94)00075-u.

Maas, C. J., \& Hox, J. J. (2005). Sufficient sample sizes for multilevel modeling. Methodology, 1(3), 86-92. https://doi.org/10.1027/16142241.1.3.86.

Marchetti, I., Koster, E. H., Klinger, E., \& Alloy, L. B. (2016). Spontaneous thought and vulnerability to mood disorders: the dark side of the wandering mind. Clinical Psychological Science, 4(5), 835-857. https://doi.org/10.1177/2167702615622383.

Newby, J. M., \& Moulds, M. L. (2011). Characteristics of intrusive memories in a community sample of depressed, recovered depressed and never-depressed individuals. Behaviour Research and Therapy, 49(4), 234-243. https://doi.org/10.1016/j.brat.2011.01.003.

Nolen-Hoeksema, S. (2000). The role of rumination in depressive disorders and mixed anxiety/depressive symptoms. Journal of Abnormal Psychology, 109(3), 504-511. https://doi.org/10.1037/0021-843X. 109.3.504.

Nolen-Hoeksema, S., \& Morrow, J. (1991). A prospective study of depression and posttraumatic stress symptoms after a natural disaster: the 1989 Loma Prieta Earthquake. Journal of Personality and Social Psychology, 61(1), 115-121. https://doi.org/10.1037/0022-3514.61. 1.115 .

Nolen-Hoeksema, S., Morrow, J., \& Fredrickson, B. L. (1993). Response styles and the duration of episodes of depressed mood. Journal of Abnormal Psychology, 102(1), 20-28. https://doi.org/10.1037/ 0021-843X.102.1.20.
Nolen-Hoeksema, S., Wisco, B. E., \& Lyubomirsky, S. (2008). Rethinking rumination. Perspectives on Psychological Science, 3(5), 400-424. https://doi.org/10.1111/j.1745-6924.2008.00088.x.

Ong, A. D., Bergeman, C. S., Bisconti, T. L., \& Wallace, K. A. (2006). Psychological resilience, positive emotions, and successful adaptation to stress in later life. Journal of Personality and Social Psychology, 91(4), 730-749. https://doi.org/10.1037/0022-3514. 91.4.730.

Petrocchi, N., \& Ottaviani, C. (2016). Mindfulness facets distinctively predict depressive symptoms after two years: the mediating role of rumination. Personality and Individual Differences, 93, 92-96. https://doi.org/10.1016/j.paid.2015.08.017.

Preacher, K. J., Curran, P. J., \& Bauer, D. J., (2003). Simple intercepts, simple slopes, and regions of significance in HLM 2-way interactions. https://www.quantpsy.org/interact/hlm2.htm. Accessed 7 July 2020.

Rasmussen, A. S., Johannessen, K. B., \& Berntsen, D. (2014). Ways of sampling voluntary and involuntary autobiographical memories in daily life. Consciousness and Cognition, 30, 156-168. https://doi. org/10.1016/j.concog.2014.09.008.

Rude, S. S., Wenzlaff, R. M., Gibbs, B., Vane, J., \& Whitney, T. (2002). Negative processing biases predict subsequent depressive symptoms. Cognition \& Emotion, 16(3), 423-440. https://doi.org/10. 1080/02699930143000554.

Schmeichel, B. J., Volokhov, R. N., \& Demaree, H. A. (2008). Working memory capacity and the self-regulation of emotional expression and experience. Journal of Personality and Social Psychology, 95(6), 1526-1540. https://doi.org/10.1037/a0013345.

Schoofs, H., Hermans, D., \& Raes, F. (2010). Brooding and reflection as subtypes of rumination: evidence from confirmatory factor analysis in nonclinical samples using the Dutch Ruminative Response Scale. Journal of Psychopathology and Behavioral Assessment, 32(4), 609-617. https://doi.org/10.1007/s10862-010-9182-9.

Segal, Z. V., Williams, M., \& Teasdale, J. (2002). Mindfulness-based cognitive therapy for depression. New York: The Guildford Press.

Sheehan, D. V., Lecrubier, Y., Sheehan, K. H., Amorim, P., Janavs, J., Weiller, E., Hergueta, T., Baker, R., \& Dunbar, G. C. (1998). The Mini-International Neuropsychiatric Interview (M.I.N.I): the development and validation of a structured diagnostic psychiatric interview for DSM-IV and ICD-10. The Journal of Clinical Psychiatry, 59(20), 22-33.

Smets, J., Wessel, I., Schreurs, E., \& Raes, F. (2012). The interplay between rumination and intrusions in the prediction of concurrent and prospective depressive symptoms in two nonclinical samples. The Psychological Record, 62(4), 777-788. https://doi.org/10.1007/ BF03395835.

Teasdale, J. D. (1988). Cognitive vulnerability to persistent depression. Cognition \& Emotion, 2(3), 247-274. https://doi.org/10.1080/ 02699938808410927.

Thompson, B. L., \& Waltz, J. (2010). Mindfulness and experiential avoidance as predictors of posttraumatic stress disorder avoidance symptom severity. Journal of Anxiety Disorders, 24(4), 409-415. https://doi.org/10.1016/j.janxdis.2010.02.005.

Treynor, W., Gonzalez, R., \& Nolen-Hoeksema, S. (2003). Rumination reconsidered: a psychometric analysis. Cognitive Therapy Research in Higher Education, 27(3), 247-259. https://doi.org/10.1023/A: 1023910315561.

Valentine, E. R., \& Sweet, P. L. (1999). Meditation and attention: a comparison of the effects of concentrative and mindfulness meditation on sustained attention. Mental Health, Religion and Culture, 2(1), 59-70. https://doi.org/10.1080/13674679908406332.

Watkins, E. R., \& Moulds, M. L. (2009). Thought control strategies, thought suppression, and rumination in depression. International Journal of Cognitive Therapy, 2(3), 235-251. https://doi.org/10. 1521/ijct.2009.2.3.235. 
Watson, L. A., Berntsen, D., Kuyken, W., \& Watkins, E. R. (2012). The characteristics of involuntary and voluntary autobiographical memories in depressed and never depressed individuals. Consciousness and Cognition, 21(3), 1382-1392. https://doi.org/10.1016/j.concog. 2012.06.016.

Wegner, \& Zanakos. (1994). Chronic thought suppression. Journal of Personality, 62(4), 615-640. https://doi.org/10.1111/j.1467-6494. 1994.tb00311.x.

Wegner, D. M., Schneider, D. J., Carter, S. R., \& White, T. L. (1987). Paradoxical effects of thought suppression. Journal of Personality and Social Psychology, 53(1), 5-13. https://doi.org/10.1037//00223514.53.1.5.

Wenzlaff, R. M., Meier, J., \& Salas, D. M. (2002). Thought suppression and memory biases during and after depressive moods. Cognition \& Emotion, 16(3), 403-422. https://doi.org/10.1080/ 02699930143000545 .
Wilkinson, P. O., \& Goodyer, I. M. (2006). Attention difficulties and mood-related ruminative response style in adolescents with unipolar depression. Journal of Child Psychology and Psychiatry, 47(12), 1284-1291. https://doi.org/10.1111/j.1469-7610.2006.01660.x.

Williams, A. D., \& Moulds, M. L. (2007). An investigation of the cognitive and experiential features of intrusive memories in depression. Memory, 15(8), 912-920. https://doi.org/10.1080/ 09658210701508369.

Zahniser, E., \& Conley, C. S. (2018). Interactions of emotion regulation and perceived stress in predicting emerging adults' subsequent internalizing symptoms. Motivation and Emotion, 42(5), 763-773. https://doi.org/10.1007/s11031-018-9696-0.

Publisher's Note Springer Nature remains neutral with regard to jurisdictional claims in published maps and institutional affiliations. 